九州大学学術情報リポジトリ

Kyushu University Institutional Repository

\title{
A FURTHER COMPARISON OF TWO-SAMPLE NONPARAMETRIC TESTS FOR DISPERISON I
}

Yanagawa, Takashi

Department of General Education, Kumamoto University

https://doi.org/10.5109/13041

出版情報 : 統計数理研究. 14 (1/2), pp. 15-24，1970-03. Research Association of Statistical Sciences バージョン :

権利関係 : 


\title{
A FURTHER COMPARISON OF TWO-SAMPLE NONPARAMETRIC TESTS FOR DISPERSION I
}

By

\author{
Takashi YanagaWA*
}

(Received January 12, 1970)

\section{Summary}

This paper is concerned with two-sample nonparametric tests for dispersion alternatives. Various nonparametric tests such as Tamura's $Q$, Sukhatme's $T$, Mood's $M$ and Freund-Ansari's $W$ (Barton-David's and Siegel-Tukey's) are considered and compared from the point of view of the Bahadur asymptotic efficiency for the symmetric distribution. Final results are given in section 4 .

\section{Introduction}

Let $X_{1}, X_{2}, \cdots, X_{m}$ and $Y_{1}, Y_{2}, \cdots, Y_{n}$ be two samples of independent observations drawn from two populations with cumulative distribution functions $F\left((x-\xi) / \sigma_{1}\right)$ and $F\left((x-\eta) / \sigma_{2}\right)$, respectively. We shall assume in what follows that $F(x)$ is absolutely continuous and $F(0)=1 / 2$.

For testing the hypotheses

$$
\left\{\begin{array}{l}
H_{0}: \sigma_{1}=\sigma_{2} \\
A H: \sigma_{1} \neq \sigma_{2} \quad\left(\text { or } \sigma_{1}<\sigma_{2}\right),
\end{array}\right.
$$

various attempts have been made to construct nonparametric tests by authors such as Mood [9], Sukhatme [11], Freund and Ansari [7], Barton and David [4], Tamura [12], Siegel and Tukey [10], Capon [5], Klotz [8] and others. But as Klotz [8] has pointed out, there is (at least asymptotically) an equivalence in the sense of test statistics among the test of Freund-Ansari, Barton-David and Siegel-Turkey, and hence we shall restrict our consideration to the tests of Sukhatme, Tamura, Mood and Freund-Ansari, whose test statistics are given as follows.

Let denote $\min (x, y)$ and $\max (x, y)$ by $x \wedge y$ and $x \vee y$, respedtively.

Sukhatme's $T$ :

$$
\begin{aligned}
& T^{(1)}=\frac{1}{m n} \sum_{i=1}^{m} \sum_{j=1}^{n} \phi\left(x_{i}, y_{j}\right), \\
& T^{(2)}=\frac{1}{m n} \sum_{i=1}^{m} \sum_{j=1}^{n} \phi\left(y_{j}, x_{i}\right) .
\end{aligned}
$$

where

* Department of General Education, Kumamoto University, Kumamoto, Japan. 
Tamura's $Q$ :

$$
\phi(x, y)= \begin{cases}1 ; & \text { if } 0 \wedge y<x<0 \vee y \\ 0 ; & \text { otherwise }\end{cases}
$$

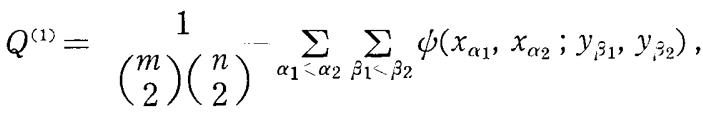

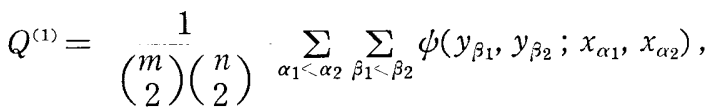

where

Mood's $M$ :

$$
\phi\left(x_{1}, x_{2} ; y_{1}, y_{2}\right)= \begin{cases}1 ; & \text { if } y_{1} \wedge y_{2}<x_{1}, x_{2}<y_{1} \vee y_{2} \\ 0 ; & \text { otherwise }\end{cases}
$$

where

$$
M=\frac{1}{N} \sum_{i=1}^{N}\left(i-\frac{N+1}{2}\right)^{2} Z_{N, i}
$$

$$
\begin{aligned}
& N=m+n, \\
& Z_{N, i}= \begin{cases}1 ; & \text { if the } i \text {-th smallest in the combined sample is an } X, \\
0 ; & \text { otherwise. }\end{cases}
\end{aligned}
$$

Freund-Ansari's $W$ :

$$
W=\frac{1}{N} \sum_{i=1}^{N}\left(\frac{N+1}{2}-\left|\frac{N+1}{2}-i\right|\right) Z_{N, i} .
$$

It is noted that the Sukhatme's $T$ and Tamura's $Q$ are $U$ statistics, while Mood's $M$ and Freund-Ansari's $W$ are rank statistics. The critical region of each test is given, respectively one sided $\left(A H: \sigma_{1}<\sigma_{2}\right)$ and two sided testing problem, by $T^{(1)}>c, T^{(2)}<c, Q^{(1)}>c, Q^{(2)}<c, M>c$ or $W>c\left|T^{(i)}\right|>c,\left|Q^{(i)}\right|>c,|M|>c$ or $|W|$ $>c, i=1,2$, where $c$ is a generic constant. The asymptotic efficiencies of these tests have been so far investigated from the point of view of the Pitman efficiency. It is known that both $T^{(1)}$ (or $T^{(2)}$ ) and $W$ have the same asymptotic efficiencies and $Q^{(1)}$ (or $Q^{(2)}$ ) and $M$ have another same asymptotic efficiencies, and the latter is larger for certain wide class of distributions.

Since the Pitman efficiency is a measure of efficiency concerning a power in the neighbourhood of $\theta=\theta_{0}$, the tests with same Pitman efficiency do not necessarily have the same efficiency for any $\theta>0$. Take Tamura's $Q^{(1)}$ and $Q^{(2)}$ for example. Though they are shown to have the same Pitman efficiency, more of our experiences and the recent paper of the author [13] shows $Q^{(1)}$ more powerful than $Q^{(2)}$ for testing problem $H: \theta=1$ vs. $A H: \theta>1$. Therefore further investigations are needed for the tests with the same Pitman efficiency.

The purpose of this paper is to give a further comparison to these tests based on the statistics mentioned above by means of the Bahadur asymptotic efficiency [2]. As noticed by Bahadur [3], Bahadur efficiency has some pitfalls, since it is an approximate measure of efficiency. To cover these pitfalls some Monte Carlo ex- 
periments are being prepared for the forth comming paper.

\section{Asymptotic slopes of tests}

We shall assume the location parameters $\xi$ and $\eta$ to be known. In the case of Tamura's $Q$, Mood's $M$ or Freund-Ansari's $W$, it is sufficient to assume only the difference $\eta-\xi$ to be known, since they are translation invariant. Since all tests under consideration are scale invariant, we can assume without loss of generality throughout the paper that two samples, $X$ of size $m$ and $Y$ of size $n$, are drawn from the population $F(x)$ and $G(x)=F(x / \theta)$, respectively, where $\theta=\sigma_{2} / \sigma_{1}$. Then the hypotheses (1.1) are reduced to

$$
\left\{\begin{array}{l}
H_{0}: \theta=1 \\
A H: \theta \neq 1 \quad(\text { or } \theta>1) .
\end{array}\right.
$$

Let $m=\rho N, n=(1-\rho) N$ and $0<\rho<1$. The asymptotic slope of the test statistic $T=T_{N}$ is defined by Bahadur [2] as follows. Let $T=\left\{T_{N}\right\}$ be a sequence of test statistics which satisfy the following conditions.

I. There exists a continuous distribution function $F$ such that under $H_{0}$

$$
\lim _{N \rightarrow \infty} P_{r}\left[T_{N} \leqq t\right]=F(t) \quad \text { for any } t .
$$

II. There exist a constant $a, 0<a<\infty$, such that

$$
\log [1-F(t)]=-\frac{a t^{2}}{2}[1+0(1)] \quad \text { as } t \rightarrow \infty .
$$

III. There exists a function $b(\theta), 0<b(\theta)<\infty$ under $A H$ such that for any $\theta \neq 1$

$$
\lim _{N \rightarrow \infty} P_{r}\left[\left|\frac{T_{N}}{\sqrt{ } N}-b(\theta)\right|>\varepsilon\right]=0 \quad \text { for any } \varepsilon>0 .
$$

Let $K_{N}(x)=-2 \log L_{N}(x)$, where $L_{N}(x)=1-F\left(T_{N}(x)\right)$. Then it can be shown that under non null hypothesis $\frac{K_{N}}{N}$ tends in probability to the quantity $C(\theta)=a[b(\theta)]^{2}$ as $N \rightarrow \infty$. The quantity $C(\theta)$ is called by Bahadur as an asymptotic slope of the test statistics $T=\left\{T_{N}\right\}$.

Now we shall give the asymptotic slope of $T^{(1)}$. Let

$$
T_{N}^{(1)}=\left(T^{(1)}-\frac{1}{4}\right) /\left(\frac{m+n}{48 m n}\right)^{1 / 2}
$$

Then under $H_{0}, T_{N}^{1}$ is known (for example see [11]) to have limiting standard normal distribution. Thus above conditions I and II are satisfied with $a=1$. By using Chebychev's inequality the quantity $b_{T^{(1)}}(\theta)$ which satisfies the condition III is easily obtained as follows.

$$
b_{T^{(1)}}(\theta)=(48 \rho(1-\rho))^{1 / 2}\left[\int_{0}^{\infty} F d G-\int_{-\infty}^{0} F d G-1 / 4\right] .
$$

Thus the asymptotic slope of $T^{(1)}$ is obtained as $C_{T^{(1)}}(\theta)=b_{T^{(1)}}^{2}(\theta)$. 
From the similar computations the asymptotic slope of each test statistic dealt with in this paper is given as follows.

For $T^{(1)}, T^{(2)}$ and $W$

$$
C_{T^{(1)}}(\theta)=C_{W}(\theta)=48 \rho(1-\rho)\left[\int_{0}^{\infty} F d G-\int_{-\infty}^{0} F d G-1 / 4\right]^{2},
$$

for $Q^{(1)}$ and $Q^{(2)}$

$$
C_{T^{(2)}}(\theta)=48 \rho(1-\rho)\left[\int_{0}^{\infty} G d F-\int_{-\infty}^{0} G d F-1 / 4\right]^{2} ;
$$

and for $M$

$$
C_{M}(\theta)=180 \rho(1-\rho)\left[(1-2 \rho) / 6-2(1-\rho) \int_{-\infty}^{\infty} F G d G-\rho \int_{-\infty}^{\infty} F^{2} d G+\int_{-\infty}^{\infty} F d G\right]^{2} .
$$

REMARK: $M$ and $W$ are rank statistics of Chernoff-Savage type. In general, under some regurality conditions given by Chernoff-Savage [6], asymptotic slopes of the rank tests of Chernoff-Savage type are given as follows;

where

$$
C(\theta)=\left(\mu-\mu_{0}\right)^{2} / \tau^{2}
$$

$$
\begin{aligned}
\mu & =\int_{-\infty}^{\infty} J[H(x)] d F(x), \\
\mu_{0} & =\int_{0}^{1} J(x) d x, \\
\tau^{2} & =\frac{1-\rho}{\rho}\left[\int_{0}^{1} J(x) d x-\left(\int_{0}^{1} J(x) d x\right)^{2}\right],
\end{aligned}
$$

$H(x)$ and $J(x)$ are defined in Chernoff-Savage [6].

Let $\mathscr{F}_{s}$ be the class of distribution functions which are absolutely continuous and symmetric about the origin.

We need the following lemma in section 3 .

LemmA. Let $F(x) \in \mathscr{F}_{8}$ and $G(x)=F(x / \theta)$, then

$$
\begin{array}{ll}
1 / 4<\int_{-\infty}^{\infty} F^{2} d G \leqq 1 / 3 & \text { for } 0<\theta \leqq 1, \\
1 / 3<\int_{-\infty}^{\infty} F^{2} d G \leqq 1 / 2 & \text { for } 1<\theta<\infty ;
\end{array}
$$

$$
1 / 4<\int_{-\infty}^{\infty} F G d G \leqq 1 / 3 \quad \text { for } 0<\theta \leqq 1 \text {, }
$$$$
1 / 3<\int_{-\infty}^{\infty} F G d G \leqq 3 / 8 \quad \text { for } 1<\theta<\infty \text {; }
$$

$$
1 / 4<\int_{0}^{\infty} F d G \leqq 3 / 8 \quad \text { for } 0<\theta \leqq 1
$$




$$
3 / 8<\int_{0}^{\infty} F d G \leqq 1 / 2 \quad \text { for } 1<\theta<\infty ;
$$

$\quad \int_{-\infty}^{\infty} F^{2} d G \leqq \int_{-\infty}^{\infty} F G d G \quad$ for $0<\theta \leqq 1$,

$$
\int_{-\infty}^{\infty} F^{2} d G>\int_{-\infty}^{\infty} F G d G \quad \text { for } 1<\theta<\infty .
$$

PROOF. Since

and

$$
\alpha(\theta) \equiv \int_{-\infty}^{\infty} F^{2} d G=1 / 2-2 \int_{0}^{\infty} F(\theta t) F(-\theta t) d F(t)
$$

$$
\alpha\left(\theta_{1}\right)-\alpha\left(\theta_{2}\right)=2 \int_{0}^{\infty}\left(F\left(\theta_{1} t\right)-F\left(\theta_{2} t\right)\right)\left(F\left(\theta_{1} t\right)+F\left(\theta_{2} t\right)-1\right) d F(t)>0
$$

for any $\theta_{1}>\theta_{2}>0$, we find that $\alpha(\theta)=\int F^{2} d G$ is an increasing function of $\theta>0$. Thus we obtain (i). (ii) is immediately obtained from (i). (iii) is obvious. By a similar argument as (i), we can easily prove (iv).

(Q.E. D.)

\section{Comparison of the tests}

Let $T_{N}^{(1)}, T_{N}^{(2)}$ be two sequences of test statistics with asymptotic slopes $C_{T_{N}^{(1)}}^{(\theta)}$ and $C_{T_{N}^{(2)}}(\theta)$, respectively, then Bahadur asymptotic efficiency of $T_{N}^{(1)}$ relative to $T_{N}^{(2)}$ is defined as $\varphi_{T_{N}^{(1)}, T_{N}^{(2)}}(\theta)=C_{T_{N}^{(1)}}(\theta) / C_{T_{N}^{(2)}}^{(\theta)}$. Now we shall consider the comparison of each test.

THEOREM 1. ( $T^{(1)}$ vs. $T^{(2)}, Q^{(1)}$ vs. $Q^{(2)}$ and $Q^{(1)}$ vs. $\left.M\right)$

(i) For arbitrary $F$,

$$
\varphi_{T^{(2)}, T^{(1)}}(\theta)=\varphi_{W, T^{(1)}}(\theta)=1 \quad \text { for any } \theta>0, \theta \neq 1 .
$$

(ii) For any $F \in \mathscr{I}_{s}$,

$$
\begin{aligned}
& \varphi_{Q^{(2)}, Q^{(1)}}(\theta)>\varphi_{M, Q^{(1)}}(\theta)>1 \quad \text { for any } 0<\theta<1 \text {, } \\
& \varphi_{Q^{(2)}, Q^{(1)}}(\theta)<\varphi_{M, Q^{(1)}}(\theta)<1 \quad \text { for any } \theta>1 \text {. }
\end{aligned}
$$

PROOF. By integration by part (i) can be immediately obtained from (2.1). (ii) First we shall consider $Q^{(1)} v s$. $M$. Since $\int_{-\infty}^{\infty} F d G=1 / 2$ for any $F \in \mathscr{I}_{s}$ and $G(x)$ $=F(x / \theta)$, thus from (2.2) and (2.3) it follows that

$$
\begin{aligned}
C_{Q^{(1)}}(\theta)-C_{M}(\theta)= & 180 \rho(1-\rho)\left\{\left(\int_{-\infty}^{\infty} F^{2} d G-1 / 3\right)^{2}-\right. \\
& \left.\left(2 \rho / 3-2(1-\rho) \int_{-\infty}^{\infty} F G d G-\rho \int_{-\infty}^{\infty} F^{2} d G\right)^{2}\right\} \\
= & 180 \rho(1-\rho) A_{1}(\theta) A_{2}(\theta),
\end{aligned}
$$

where

$$
A_{1}(\theta)=(1-\rho)\left(\int F^{2} d G-2 \int F G d G+1 / 3\right)=(1-\rho) \int(F-G)^{2} d G>0
$$

for any $\theta>0, \theta \neq 1$, 


$$
A_{2}(\theta)=(1+\rho)\left(\int F^{2} d G-1 / 3\right)+2(1-\rho)\left(\int F G d G-1 / 3\right) .
$$

Since from the lemma $A_{2}(\theta)<0(>0)$ for $0<\theta<1(1<\theta<\infty)$, we have $C_{Q^{(1)}}(\theta)-C_{M}(\theta)$ $<0(>0)$ for $0<\theta<1(1<\theta<\infty)$. Since $C_{Q^{(1)}}(\theta) \neq 0$ for any $\theta>0, \theta \neq 1$, we get the right hand side inequality. Next, from the above results, it is immediately obtained that $\varphi_{M, Q^{(2)}}(\theta)<1(>1)$ for $0<\theta<1(1<\theta<\infty)$. Thus we get the left hand side inequality.

The theorem means that the efficiency of Tamura's $Q$, unlike that of Sukhatme's $T$, depends on the direction of the alternative hypothesis; $Q^{(1)}$ is more efficient than $Q^{(2)}$ for the one sided case $A H: \theta>1$, but less efficient for $A H: \theta<1$.

In the case one might expect to get higher efficiency by adding $Q^{(1)}$ and $-Q^{(2)}$ for the two sided testing problem. The new statistic $Q^{(12)}=Q^{(1)}-Q^{(2)}$ is also a $U$ statistic given as follows.

$$
Q^{(12)}=\frac{1}{\left(\begin{array}{c}
m \\
2
\end{array}\right)\left(\begin{array}{c}
n \\
2
\end{array}\right)} \sum_{\substack{\alpha_{1}<\alpha_{2} \\
\beta_{1}<\beta_{2}}} \omega\left(x_{\alpha_{1}}, x_{\alpha_{2}} ; y_{\beta_{1}}, y_{\beta_{2}}\right),
$$

where

$$
\omega\left(x_{1}, x_{2} ; y_{1}, y_{2}\right)=\left\{\begin{aligned}
1 & \text { if } y_{1} \wedge y_{2}<x_{1}, x_{2}<y_{1} \vee y_{2} \\
-1 & \text { if } x_{1} \wedge x_{2}<y_{1}, y_{2}<x_{1} \vee x_{2} \\
0 & \text { if otherwise. }
\end{aligned}\right.
$$

Denoting the mean value of $Q^{(12)}$ by $\mu_{12}(\theta)$, then we get

$$
\mu_{12}(\theta)=2 \iint_{x<y}[F(y)-F(x)]^{2} d G(x) d G(y)-2 \iint_{x<y}[G(y)-G(x)]^{2} d F(x) d F(y) .
$$

From the general theory of $U$ statistics $N^{1 / 2}\left(Q^{(12)}-\mu_{12}(\theta)\right)$ has limiting normal distribution with mean 0 and its asymptotic variance under null hypothesis is easily calculated as follows.

$$
\sigma_{12}^{2}(1)=\frac{4}{45 \rho(1-\rho)} \text {. }
$$

Thus the efficacy of $Q^{(12)}$ in the Mood's sense is given by

$$
\left(\left.\frac{d \mu_{12}(\theta)}{d \theta}\right|_{\theta=1} / \sigma_{12}(1)\right)^{2}=180 \rho(1-\rho)\left[\int_{-\infty}^{\infty}(2 F(x)-1) x f(x) d F(x)\right]^{2},
$$

which is equivalent to that of $Q^{(1)}$. Thus we get the following theorem.

THEOREM 2. $Q^{(12)}$ has the same Pitman efficiency as $Q^{(1)}$.

THEOREM 3. For any $F \in \mathscr{F}_{s}$, we get

$$
\begin{array}{ll}
\varphi_{Q^{(2)}}, Q^{(12)}(\theta)>\varphi_{Q^{(12)}, Q^{(1)}}(\theta)>1 & \text { for } 0<\theta<1, \\
\varphi_{Q^{(2)}, Q^{(12)}}(\theta)<\varphi_{Q^{(12)}, Q^{(1)}}(\theta)<1 & \text { for } \theta>1 ;
\end{array}
$$

(ii) (a) if $0<\rho<1 / 2$, then $\varphi_{Q}^{(12)},{ }_{M}(\theta)<1(>1)$ for $0<\theta<1(1<\theta<\infty)$,

(b) if $0<\rho<1 / 2$, then $\varphi_{Q^{(12)}, M}(\theta)=1$ for any $\theta>0, \theta \neq 1$,

(c) if $1 / 2<\rho<1$, then $\varphi_{Q}^{(12), \mu}(\theta)>1(<1)$ for $0<\theta<1(1<\theta<\infty)$. 
Proof. The asymptotic slope of $Q^{(12)}$ is easily obtained as follows.

$$
C_{Q^{(12)}}(\theta)=45, \rho(1-\rho)\left[\int_{-\infty}^{\infty} F^{2} d G-\left(\int_{-\infty}^{\infty} F d G\right)^{2}-\int_{-\infty}^{\infty} G^{2} d F+\left(\int_{-\infty}^{\infty} G d F\right)^{2}\right]^{2} .
$$

Then we get from (2.2) and (2.3)

$$
\begin{aligned}
& C_{Q^{(1)}}(\theta)-C_{Q^{(12)}}(\theta)=45 \rho(1-\rho)\left[4\left(\int_{-\infty}^{\infty} F^{2} d G-\frac{1}{3}\right)^{2}-\left(\int_{-\infty}^{\infty} F^{2} d G-\int_{-\infty}^{\infty} G^{2} d F\right)^{2}\right] \\
& =45 \rho(1-\rho) B_{1}(\theta) B_{2}(\theta) \text {, }
\end{aligned}
$$

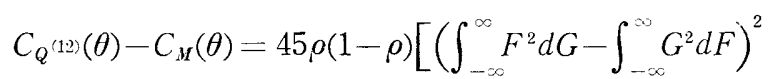

$$
\begin{aligned}
& \left.-4\left(\frac{2-\rho}{3}-2(1-\rho) \int_{-\infty}^{\infty} F G d G-\rho \int_{-\infty}^{\infty} F^{2} d G\right)^{2}\right] \\
& =45 \rho(1-\rho) D_{1}(\theta) D_{2}(\theta)
\end{aligned}
$$

for any $F \in \mathscr{F}_{s}$, where

$$
\begin{aligned}
& B_{1}(\theta)=\int_{-\infty}^{\infty} F^{2} d G+\int_{-\infty}^{\infty} G^{2} d F-2 / 3=\int_{-\infty}^{\infty}(F-G)^{2} d G, \\
& B_{2}(\theta)=3\left(\int_{-\infty}^{\infty} F^{2} d G-1 / 3\right)+2\left(\int_{-\infty}^{\infty} F G d G-1 / 3\right), \\
& D_{1}(\theta)=(1-2 \rho) \int_{-\infty}^{\infty}(F-G)^{2} d G, \\
& D_{2}(\theta)=(1+2 \rho)\left(\int_{-\infty}^{\infty} F^{2} d G-1 / 3\right)+(6-4 \rho)\left(\int_{-\infty}^{\infty} F G d G-1 / 3\right)
\end{aligned}
$$

Thus by the lemma and the similar argument as in the theorem 1 (ii) we complete the proof.

(Q. E. D.)

Since $T^{(1)}, T^{(2)}$ and $W$ are seen to have the same efficiency in the sence of Bahadur as well as Pitman, we shall in the sequel consider only $W$ among them and compare it with $Q^{(1)}$ and $M$.

Let denote by $f$ the p.d.f. of $F$, and define the class of distribution functions $\mathscr{F}_{s s}, \mathscr{F}_{s+}, \mathscr{F}_{s 0}$ and $\mathscr{F}_{s-}$ as follows.

$$
\begin{gathered}
\mathscr{F}_{s s}=\left\{F ; F \in \mathscr{F}_{s}, x f(\theta x) \text { is bounded and continuous in } \theta>0\right. \\
\quad \text { for any } x>0 \text { a. e. }(F)\}, \\
\mathscr{F}_{s+}=\left\{F ; F \in \mathscr{F}_{s s}, \int_{0}^{\infty} x f(x) F(x) d F(x)>a \int_{0}^{\infty} x f(x) d F(x)\right\}, \\
\mathscr{F}_{s 0}=\left\{F ; F \in \mathscr{F}_{s s}, \int_{0}^{\infty} x f(x) F(x) d F(x)=a \int_{0}^{\infty} x f(x) d F(x)\right\}, \\
\mathscr{F}_{s-}=\left\{F ; F \in \mathscr{F}_{s s}, \int_{0}^{\infty} x f(x) F(x) d F(x)<a \int_{0}^{\infty} x f(x) d F(x)\right\},
\end{gathered}
$$

where $a=(15+2 \sqrt{ } 15) / 30$.

THEOREM 4. ( $Q^{(1)}$ vs. $W$ and $M$ vs. $W$ ) There exists an $\varepsilon>0$ such that

(i) if $F \in \mathscr{F}_{s+}$, then $\varphi_{Q^{(1)}, W}(\theta), \varphi_{M, W}(\theta)>1$ for $1-\varepsilon<\theta<1+\varepsilon, \theta \neq 1$, 
(ii) if $F \in \mathscr{F}_{s 0}$, then both $\varphi_{Q^{(1)}, W}(\theta)-1$ and $\varphi_{M, W}(\theta)-1$ have the same sign for $1-\varepsilon<\theta<1+\varepsilon, \theta \neq 1$,

(iii) if $F \in \mathscr{F}_{s-}$, then $\varphi_{Q^{(1)}, W}(\theta), \varphi_{M, W}(\theta)>1$ for $1-\varepsilon<\theta<1+\varepsilon, \theta \neq 1$.

Proof. We shall first prove for $Q^{(1)}$ vs. $W$. From (2.1) and (2.2) it follows for any $F \in \mathscr{F}_{s}$

$$
C_{Q^{(1)}}(\theta)-C_{W}(\theta)=4 \rho(1-\rho) E_{1}(\theta) E_{2}(\theta)
$$

where

$$
\begin{aligned}
& E_{1}(\theta)=3 \sqrt{5}\left(\int_{-\infty}^{\infty} F^{2} d G-1 / 3\right)+4 \sqrt{3}\left(\int_{-\infty}^{\infty} F d G-3 / 8\right), \\
& E_{2}(\theta)=3 \sqrt{5}\left(\int_{-\infty}^{\infty} F^{2} d G-1 / 3\right)-4 \sqrt{3}\left(\int_{-\infty}^{\infty} F d G-3 / 8\right) .
\end{aligned}
$$

From the lemma mentioned above we get $E_{1}(\theta)<0(>0)$ for $0<\theta<1(1<\theta<\infty)$. Further it follows that

$$
E_{2}(\theta)=6 \sqrt{5} \int_{0}^{\infty}\left[\begin{array}{c}
F^{2}(\theta x)-2 \sqrt{3}+3 \sqrt{5} \\
3 \sqrt{5}
\end{array} F(\theta x)+\begin{array}{c}
2 \sqrt{5}+3 \sqrt{3} \\
7 \sqrt{5}
\end{array} F^{2}(x)\right] d F(x) .
$$

Thus we get $d E_{2}(\theta) / d \theta=12 \sqrt{5} A(\theta)$ for any $F \in \mathscr{F}_{s s}$, where

$$
A(\theta)=\int_{0}^{\infty} x f(\theta x)\left[F(\theta x)-\frac{3 \sqrt{5}+2 \sqrt{3}}{6 \sqrt{5}}\right] d F(x) .
$$

Since $E_{2}(1)=0$, then there exists an $\varepsilon>0$ such that (i) if $A(1)>0$, then $E_{2}(\theta)<0(>0)$ for $1-\varepsilon<\theta<1(1<\theta<1+\varepsilon)$, (ii) if $A(1)=0$, then either $E_{2}(\theta)>0$ or $<0$ for $1-\varepsilon<\theta$ $<1+\varepsilon, \theta \neq 1$ and (iii) if $A(1)<0$, then $E_{2}(\theta)>0(<0)$ for $1-\varepsilon<\theta<1(1<\theta<1+\varepsilon)$. Thus it follows that (i) if $F \in \mathscr{F}_{s+}$, then $C_{Q}(1)(\theta)-C_{W}(\theta)>0$ for $1-\varepsilon<\theta<1+\varepsilon, \theta \neq 1$, (ii) if $F \in \mathscr{F}_{s 0}, C_{Q^{(1)}}(\theta)-C_{W}(\theta)$ has the same $\operatorname{sign}$ for $1-\varepsilon<\theta<1+\varepsilon, \theta \neq 1$ and (iii) if $F \in \mathscr{F}_{s-}$, then $C_{Q^{(1)}}(\theta)-C_{W}(\theta)<0$ for $1-\varepsilon<\theta<1+\varepsilon, \theta \neq 1$. But as $C_{W}(\theta) \neq 0$ for any $\theta>0, \theta \neq 1$, thus we get the conclusions stated in the theorem. Next we shall prove for $M$ vs. $W$. From (2.1) and (2.3) it follows that for any $F \in \mathscr{F}_{s}$,

$$
C_{M}(\theta)-C_{W}(\theta)=12 \rho(1-\rho) E_{1}^{*}(\theta) E_{2}^{*}(\theta),
$$

where

$$
\begin{aligned}
& E_{1}^{*}(\theta)=\sqrt{15}\left[(\rho-2) / 3+2(1-\rho) \int_{-\infty}^{\infty} F G d G+\rho \int_{-\infty}^{\infty} F^{2} d G\right]+4\left(\int_{0}^{\infty} F d G-3 / 8\right), \\
& E_{2}^{*}(\theta)=\sqrt{15}\left[(\rho-2) / 3+2(1-\rho) \int_{-\infty}^{\infty} F G d G+\rho \int_{-\infty}^{\infty} F^{2} d G\right]-4\left(\int_{0}^{\infty} F d G-3 / 8\right) .
\end{aligned}
$$

Thus the similar computations as above lead the proof of the theorem. (Q. E. D.)
(n)

REMARK. The class of distribution functions $\mathscr{F}_{s+}$ is wide enough to include normal, logistic and double exponential distributions among others, while $\mathscr{I}_{s-}$, generally, includes distributions more spread than those belonging to $\mathscr{F}_{s+}$, Cauchy distribution for example.

\section{Conclusions}

From the theorems above we can summarise our results briefly as follows. It is noted that the comparison depends not only on the underlying distribution but 
also on the direction of the alternative hypothesis.

For the right-sided testing problem $H_{0}: \theta=1$ vs. $A H: \theta>1, Q^{(1)}$ is always the most efficient among $Q^{(1)}, Q^{(2)}, Q^{(12)}$ and $M$ for any distribution $F \in \mathscr{F}_{s}$.

For two-sided hypothesis $H_{0}: \theta=1$ vs. $A H: \theta \neq 1$, unlike Sukhatme's $T^{(1)}$ and $T^{(2)}$ have the same asymptotic efficiencies for any distributions and for any $\theta>0, \theta \neq 1$, efficiencies of $Q^{(1)}$ and $Q^{(2)}$ depend on the alternative hypothesis. Theorem 2 and 3 lead one to the following comparisons among $Q^{(1)}, Q^{(2)}, Q^{(12)}$ and $M$.

(i) For $0<\rho<1 / 2$

$$
\begin{array}{ll}
\varphi_{Q^{(2)}, Q^{(1)}}(\theta)>\varphi_{M, Q^{(1)}}(\theta)>\varphi_{Q^{(12)}, Q^{(1)}}(\theta)>1 & \text { for any } 0<\theta<1, \\
\varphi_{Q^{(2)}, Q^{(1)}}(\theta)<\varphi_{M, Q^{(1)}}(\theta)<\varphi_{Q^{(12)}, Q^{(1)}}(\theta)<1 & \text { for any } \theta>1 ;
\end{array}
$$

(ii) For $\rho=1 / 2$

$$
\begin{array}{ll}
\varphi_{Q^{(2)}, Q^{(1)}}(\theta)>\varphi_{M, Q^{(1)}}(\theta)=\varphi_{Q^{(12)}, Q^{(1)}}(\theta)>1 & \text { for any } 0<\theta<1, \\
\varphi_{Q^{(2)}, Q^{(1)}}(\theta)<\varphi_{M, Q^{(1)}}(\theta)=\varphi_{Q^{(12)}, Q^{(1)}}(\theta)<1 & \text { for any } \theta>1 ;
\end{array}
$$

(iii) For $1 / 2<\rho<1$

$$
\begin{aligned}
& \varphi_{Q^{(2)}, Q^{(1)}}(\theta)>\varphi_{Q^{(12)}, Q^{(1)}}(\theta)>\varphi_{M, Q^{(1)}}(\theta)>1 \quad \text { for any } 0<\theta<1 \text {, } \\
& \varphi_{Q^{(2)}, Q^{(1)}}(\theta)<\varphi_{Q^{(12)}, Q^{(1)}}(\theta)<\varphi_{M, Q^{(1)}}(\theta)<1 \quad \text { for any } \theta>1 \text {. }
\end{aligned}
$$

Thus within our present knowledge, we have no decisive choice among $Q^{(1)}, Q^{(2)}$, $Q^{(12)}$ and $M$ for two-side testing problems.

For any distribution belonging to $\mathscr{I}_{s+\cdots}, Q^{(1)}$ and $M$ are more efficient than $W$, but less efficient for $\mathscr{F}_{s-\text {. }}$

\section{Acknowledgements}

This work has been done while the author was in Osaka University. He express his hearty thanks to Professor M. Okamoto, Osaka University for his generous help and critical comments, especially the statistic $Q^{(12)}$ was suggested by him. The author also express his hearty thanks to Professor A. Kudô, Kyushu University for his kind discussions about the paper and pointing out several mistakes.

\section{References}

[1] Ansari, A.R. and Bradley, R. A. (1960). Rank-sum tests for dispersions. Ann. Math. Statist. 31, 1174-1189.

[2] Bahadur, R. R. (1960). Stochastic comparison of tests. Ann. Math. Statist. 31, $276-295$.

[3] BAHAdur, R. R. (1967). Rates of convergence of estimates and test statistics. Ann. Math. Statist. 38, 303-324.

[4] Barton, D. E. and David, F.N. (1958). A test for birth-order effects. Ann. Eugenics 22, 250-257.

[5] Capon, J. (1961). Asymptotic efficiency of certain locally most powerful rank tests. Ann. Math. Statist. 32, 88-100.

[6] Chernoff, H. and Savage, I. R. (1958). Asymptotic normality and efficiency of certain nonparametric test statistics. Ann. Math. Statist. 29, 972-994.

[7] Freund, J.E. and Ansari, A.R. (1957). Two-way rank sum test for variance. Virginia 
Polytechnic Institute Report supported by the office of Ordnance Research and National Science Foundation.

[8] Klotz, J.H. (1962). Nonparametric tests for scale. Ann. Math. Statist. 33, 498-512.

[9] Mood, A.M. (1954). On the asymptotic efficiency of certain nonparametric two-sample tests. Ann. Math. Statist. 25, 514-522.

10] Siegel, S. and Tukey, J.W. (1960). A nonparametric sum of rank procedure for relative spread in unpaired samples. J. Amer. Stat. Assoc. 55, 429-445.

[11] Suknatme, B. V. (1957). On certain two-sample nonparametric tests for variances. Ann. Math. Statist. 28, 188-194.

12 Tamura, R. (1960). On the nonparametric tests based on certain $U$-statiatics. Bull. Math. Statist. 9, 61-67.

[13: YaNAGAwA, T. (1970). A note on Tamura's Q. Bull. Math. Statist. 14, 25-30. 